\title{
Beiträge zur pathologischen Anatomie des Auges.
}

\author{
Von
}

Dr. E. Berger,

Privat-Docenton an der Universität in Graz.

Hierzu Tafel IV.

I. Anatomische Untersuchung eines Falles von Tenonitis.

Von 0. Ferral ${ }^{*}$ ) wurde zuerst eine Form der Entzündung des retrobulbären Gewebes beschrieben, boi welcher der entzündliche Process vorwiegend auf die Tenon'sche Kapsel beschränkt ist und es zur Ansammlung von Eitormassen innerhalb derselben kommen soll. Die von 0 . Forral angegebenen klinischen Erschoinungen stimmen in Allgemeinen mit denen der Fntzündung des retrobulbären Zellgewebes überein. Auch die von 0 . Ferral angegebenen differential-diagnostischen Symptome: Unempfindlichkeit des Knochens gegen Druck und das Beschränktsein des extzündlichen Oedems des oberen Lides auf seinen Tursaltheil lassen die Annahme von Tenonitis nicht genügend gesichert erscheinen. Den anatomischen Nachweis dieser Erkrankung hat 0 . Ferral nicht geliefert.

Mackenzie $*$ glaubte, dass im zweiten Stadium der Panophthalmitis sich Eiteransammlung in der Tenon'schen Kapsel findet.

*) Dublin Journal of med. science. 1841. Vol. XIX., p. 343.

**) Traité pratique des maladies des yeux. tioms ed. (T'rad. par Warlomont et Testelin) 1856. I., p. 430. 
Nach Schweigger*) pflegt bei allen Entzündungen des retrobulbären Zellgewebes die Tenon'sche Kapsel ebenfalls entzündlich afficirt zu sein.

Berlin **) spricht sich mit Entschiedenheit dagegen aus, dass es zu einer Eiteransammlung innerhalb der Tenon'schen Kapsel komme, wie dies namentlich von O. Ferral angenommen wurde. "Wer je in der Lage war, einen Bulbus im Zustande beginnender Panophthalmitis oder der bestehenden Phlegmone orbitze ${ }^{* * *}$ ) zu enucleiren, wird sich im Gegentheil von dem festen Zusammenhang der Kapsel und des Augapfels, welche unter dem Einflusse der Entzündung zu Stande kommt, überzeugt haben."

Auf das gleiche Verhalten der Tenon'schen Kapsel bei Panophthalmitis wurde zuerst von v, Graefe $\dagger$ ) aufmerksam gemacht.

Förster $\dagger \dagger$ ) findet bei der Section eines Falles von Sinusthrombose „die Tenon'sche Kapsel serös infiltrirt, verdickt und aufgelockert". Eine Eiteransammlung innerhalb der letzteren wurde nicht beobachtet.

Linhart $\dagger+\dagger$ ), welcher Untersuchungen über die anatomisch-physiologischen Eigenschaften der Tenon'schen Kapsel machte, denkt sich das Exsudat als seröses und stellt folgendes Krankheitsbild auf: 1. mässiger Exophthalmus; 2. die Bewegungen des Auges sind langsam, schmerzhaft, nicht beschränkt; 3. wird der Bulbus gegen die Orbita gedrückt, so erscheint die Umgebung desselben gespannt und ,undeutlich fluctuirend".

In ähnlicher Weise wird das Krankheitsbild von von Wecker*) geschildert.

Bei v. Mooren $* * \dagger)$ finden wir folgende Schilderung: „Eine gehinderte Beweglichkeit des Bulbus mit leichter Vor-

*) Handbuch der spec. Augenheilkunde. p. 184.

**) Graefe u. Saemisch, Handbuch, VI. p. 535.

***) Berlin, Klin. Monatsbl. 1866. p. 85.

†) Arch. f. Ophthalm. III. 2, p. 421.

††) Arch. f. Ophthalm. XIV. 1, p. 222.

††) Bemerkungen über die Tenon'sche Kapsel. Verhandl. d. phys.-med. Ges. zu Würzburg. IX., p. 245.

*) Traité theoretique et pratique des maladies des yeux. II. ed. I., p. 762.

**) Ophthalmolog. Mittheilungen. 1874. p. 13. 
wärtsdrängung des Auges und durchsichtiger, chemotischer Auftreibung der Bindehaut bilden eine Reihe von Erscheinungen, welche charakteristisch für das Auftreten von Tenonitis sind."

Ueber die klinischen Erscheinungen von Tenonitis berichten ferner noch Soelberg-Wells *), Friedberg***), Fano***), Bull $\dagger$ ), v. Schiess-Gemuseus $\dagger \dagger$ ), Heyl $\dagger+\dagger$ ), Rampoldi*广) und Hock.**广)

Allein sämmtliche oben genannten Autoren theilen keine Beobachtungen über den pathologisch-anatomischen Befund mit. Mit Recht sagt daher Berlin; „Fragen wir nach dém pathologisch-anatomischen Befunde, so treffen wir sofort auf die schwache Seite dieses Krankheitsbildes." Linhart erklärt, dass die Tenotinitis "eben nur eine hingestellte Möglichkeit ist."

Abgesehen von Panophthalmitis und Orbitalphlegmone findet man Erkältung als veranlassendes Moment der Entzündung der Tenon'schen Kapsel angegeben; ferner wird letztere veranlasst durch Schieloperationen. v. Wecker beobachtete Tenonitis 1 mal bei 600 Schieloperationen, v. Mooren 5 mal bei 3705, Bull 3 mal nach der Intenotomie. Heyl fand in einem Fall metastatisches Auftreten von Tenonitis bei Diphtherie.

Aus obiger Darstellung ergiebt sich, dass die Veroffentlichung eines Falles, in welchem der Obductionsbefund den Nachweis von Tenonitis liefert, immerhin gerechtfertigt erscheinen mag.

*) Treatise of the diseases of the eye. p. 633.1869.

**) Virchow's Archiv XXX., p. 585.

***) Traité pratique des maladies des yeux. 1866. I., p. 128.

t) Med. Record. 1. Novbr, 1871.

††) Zur Lehre von der Tenonitis. Fünf Fälle von Tenonitis. Klin. Monatsbl. 1878. p. 305.

$+\dagger$ ) Ueber metastatische Tenonitis bei Diphtherie. Americ. Journ. CLVIII, p. 429.

${ }^{*}$ ) Uno caso di Tenonile primativa. Ann. d'Oftalm. May XI. p. 170 .

**+) Bericht der Privat-Augenheilanstalt. Wien. med. Bl. 1883. p. $12-16$. 
Durch die Güte des Herrn Dr. O. Eberstaller, Assistenten an der anatomischen Lehrkanzel in Graz gelangte ich in den Besitz eines phthisischen Bulbus, welcher mit den Orbitalgebilden aus der Orbita herauspräparirt wurde. Derselbe stammte von der Leiche eines alten weiblichen Cretins aus dem Knittelfelder Siechenhause. Herr Dr. Eb erstaller hatte die Freundlichkeit sich über die der Phthisis bulbi zu Grunde liegenden Momente zu erkundigen, es konnte derselbe jedoch hierüber keinen befriedigenden Aufschluss erhalten.

Als ich den Bulbus aus der Tenon'schen Kapsel, ähnlich wie bei den Enucleationen, herauspräparirte, war der Umstand auffallend, dass dies nur sehr schwer gelang. Es zeigte sich, dass zwischen dem inneren und dem äusseren Blatte der Tenon'schen Kapsel eine faserig-gallertige Nasse von der Dicke von $1^{1 / 2}-2^{\prime \prime}$ abgelagert war, welche bei dem Herauspräpariren des Bulbus theils an dessen Oberfläche, theils an dem äusseren Blatte der Tenon'schen Kapsel haften blieb. Einzelne faserige Stränge leisteten bei der Losschälung des Auges einen stärkeren Widerstand.

Ueber die Ausbreitung dieser faserigen Massen an der Oberfläche des Augapfels konnte ich folgendes feststellen. Es beginnen dieselben 3-4 Mm. hinter dem Corneoscleralrande und bedecken seine Oberfläche bis in die Nähe der Eintrittsstelle des Sehnerven.

Das Auge bot hochgradige Veränderungen. Die Cornea ist auf ein kleines wenige Millimeter grosses Stück geschrumpft. Die Sclerotica erscheint sehr stark gefaltet.

Das Hornhautepithel ist verdickt, zeigt papillenartige Vertiefungen. Die Substantia propia der Hornhant ist von Gefässen durchsetzt. Die Descemet'sche Membran ist stark gefaltet und lässt sich leicht beim Zerzupfen des Präparates in Lamellen spalten, wie dies bei den entzündlichen Processen der Hornhaut bekanntlich häufig der Fall 
ist. Es ist diese Erscheinung darauf zurückzuführen, dass die zwisehen den Lamellen der M. Descemeti vorhandene Kittsubstanz durch den entzündlichen Vorgang verändert wird.

Die vordere Kammer ist kaum von der Grösse eines Hirsekornes, theilweise mit einer feinkörnigen Masse, in welcher Rundzellen sich finden (serös eitriges Exsudat), erfüllt.

Die Iris ist bindegewebig entartet und unterscheidet sich durch ibren Reichthum an Pigment von der ihre Vorderfäcche bedeckenden bindegewebigen Schwarte. Die Pupille ist nicht vorhanden. Die Linse fehit.

Der Ciliarkörper und die Aderhaut sind von der Sclerotica abgehoben. Der zwischen denselben gelegene Raum ist von bindegewebigen Massen, die ron zahlreichen Gefässen durchsetzt sind, und schwarzes Pigment theils in Form von Körnchen, theils diffus, theils in sternförmigen Pigmentzellen enthalten, erfüllt. Es ist dieses Bindegewebe durch Organisation eines zwischen der Aderhaut und der Selerotica abgelagerten Exsudates entstanden. Es haben diese bindegewebigen Massen eine solche Dicke erlangt, dass die Ciliarfortsätze sich nahezu berühren und die Aderhaut auf der einen Seite des Meridionalschnittes durch den Bulbus $4 \mathrm{~mm}$ von der Sclerotica absteht. Zahlreiche, theils grössere, theils kleinere cystenartige Hohlräume finden sich zwischen diesen neugebildeten Massen.

Es sind diese Exsudate, welche zwischen der Aderhaut und der Sclerotica abgelagert sind und in ein bindegewebiges Maschenwerk sich umwandeln, bereits von Iwanoff, Knapp, Hirschberg u. A. beschrieben worden. Nach Brailey und Lobo*) sollen diese Bindegewebsbil-

*) Ueber patholog. Neubildung in der Aderhant. Centralbl. f. Augenheilk. 1882, p. 260. 
dungen durch Verdickungen der M. Suprachorioidea zu Stande kommen. Goldzieher *) hebt die Analogie zwischen diesen Bindegewebsbildungen und den pleuritischen sowie peritonitischen Schwarten hervor. Derselbe schlägt vor, die denselben zu Grunde liegende Erkrankung als Chorjoiditis externa zu bezeichnen.

Die Pars ciliaris retinae ist beträchtlich verdickt, mit kolbenartigen Fortsătzen versehen, welche in die cyclitischen Schwarten und in den Glaskörper hineinragen. Es sind dieselben theilweise von Pigment umscheidet. Von v. Schiess-Gemuseus wurden dieselben zuerst beschrieben und als neugebildete Gefässe richtig erkannt.

Der Glaskorper ist bindegewebig entartet, zwischen den Fasermassen desselben finden sich zahlreiche Kerne und Anhäufungen von Rundzellen. An einzelnen Stellen der Sclerotica konnte ich ebenfalls streifenformig angeordnete Rundzellen nachweisen, ferner finde ich dieselben in diesem Falle in der Umgebung der Austrittsstelle der Venae vorticosae Stenonis, woselbst auch Kerne zwischen den Fasern der Sclerotica sich angehäuft finden. Einen ähnlichen Befund habe ich bei einem an Iridocyclitis erkrankten Auge gesehen, welches wegen drehender sympathischer. Affection enucleirt wurde.

Nach den Untersuchungen von Schwalbe **) wird die im suprachorioidalen Lymphraume angesammelte Lymphe durch Lymphwege, welche neben den Venae vorticosae verlaufen, in den Tenon'schen Raum abgeleitet. Obige Infiltration mit Rundzellen, von welcher ich vorläufig noch dahingestellt lassen muss, ob sie ein bei Iridocyclitis häufiger Befund ist, mag wohl den Abfluss der Lymphe wenn nicht vollständig verhindern, so doch

*) Ueber Schwartenbildungen in der Suprachoroidea als Product einer Chorioiditis externa. Centralbl. f. Augenheilk. 1883, p. 41; Therapie der Augenkrankheiten 1882, p. 113.

**) Stricker's Handbuch der Gewebelehre, p. 1065. 
wesentlich stören und auf diese Weise mit zur Frhőhung des intraoculären Druckes beitragen.

Die abgeloste Netzhaut ist bindegewebig entartet. In der Umgebung der Centralgefässe finden sich sowohl in der Netzhaut, als auch im Sehnerven sternformige Pigmetzellen eingelagert. In der Nähe des Sehnerven ist in der Aderbaut eine ihre inneren Schichten einnehmende Knochenneubildung zu beobachten. Die bindegewebigen Balken des Sehnerven sind beträchtlich verbreitert; die Nervenfasern sind zwischen denselben nachweisbar, besitzen jedoch keine Markscheiden. Zwischen den Scheiden des Sehnerven*) finde ich die Ablagerung eines bindegewebig organisirten Exsudates.

Das innere Blatt der 'Tenon'schen Kapsel ist, wie an Meridionalschnitten durch den Bulbus sich ergiebt, verdickt; ibre Fasern sind aufgelockert, stellenweise sind Rundzellen zwischen dieselben eingelagert.

Auf der Oberfläehe der Tenon'schen Kapsel findet sich aus welligförmig gekrümmten Fasern bestehendes Bindegewebe mit zahlreichen eingelagerten länglichen bis spindelförmigen Kernen und einzelnen Rundzellen. Neugebildete Gefässe, welche mit den Blutgefässen der Sclerotica und des imneren Blattes der Tenon'schen Kapsel communiciren, durchsetzen das neugebildete Bindegewebe. Zwischen den Bindegewebsfasern finden sich, namentlich in der Nähe der Tenon'schen Kapsel, Anhäufungen von Endothelzellen derselben, von welchen viele 2 Kernkörperchen, mehrere 2 Kerne enthalten.

Die Sehnen der Augenmuskeln sind sowohl an ihrer äusseren, als auch an ihrer inneren dem Bulbus zugekehrten Fläche von den neugebildeten Bindegewebs-

*) Die durch Injectionsversuche nachgewiesene Communication zwischen dem intervaginalen Lymphraume des Sehnerven und dem suprachorioidealen Lymphraume mag für die Fortpflanzung der Entzïndung von dem einen in den anderen von Bedeutung sein. 
massen umgeben. Im Gewebe der Sehnen selbst lässt sich keine Veränderung erkennen.

Auf der äusseren Oberfläche der Sehne eines Augenmuskels, finde ich eine kleine Anhäufung von Pigmentzellen, eingeschlossen in eine bindegewebige Hülle. Diese kleine Geschwulst ist mit bindegewebigen Fasern und zahlreichen Capillargefässen durchsetzt.

Meridionalschnitte durch den hinteren Theil des Bulbus lassen über die Ausbreitung der neugebildeten bindegewebigen Massen erkennen, dass dieselben bis in die Nähe des Sehnerven reichen und sich in dem supravaginalen Raume desselben fortsetzen, welcher bekanntlich mit dem Tenon'schen Raume communicirt (Schwalbe).

Wenn wir die in diesem Falle an der Oberfláche des Bulbus sich ergebenden pathologischen Veränderungen zusammenfassen, so ergiebt sich, dass die Tenon'sche Kapsel verdickt erscheint, und dass zwischen dem inneren und dem äusseren Blatte derselben sich neugebildete bindegewebige Fasermassen finden, welche durch Organisirung eines entzündlichen Exsudates, das im T'enon'schen Raume sich ausbreitete, entstanden sind.

Der Ausgang des entzündlichen Processes ist in diesem Falle, wie dies bei den adhäsiven Formen der Pleuritis und anderen Entzündungen seröser Höhlen der Fall ist, vollständige Obliteration des Tenon'schen Raumes.

Das hinter der Tenon'schen Kapsel gelegene rebrobulbäre Zellgewebe zeigte keine entzündlichen Erscheinungen.

Wenn das Exsudat im Tenon'schen Raume ein sehr reichhaltiges sein sollte, so kann dies gewiss nicht ohne Einfluss auf die denselben durchsetzende Venae vorticosae Stenonis sein, indem dieselben ein Compression erleiden müssten. 
Leber*) fand bekanntlich bei der Unterbindung der Venae vorticosae, „neken einer sehr hochgradigen Hyperaemie des ganzen Uvealtractus eine bedeutende Steigerung des Augendruckes." Die Zunahme des intraoculären Druckes beträgt nach Adamück**) bei Katzen $90 \mathrm{Mm}$., bei Kaninchen nach Leber „das Doppelte des normalen und etwas darüber. Die Augen fühlen sich bei der Palpation steinhart an."

Wenn in wenigen der Beschreibungen der klinischen Symtome von Tenonitis einer vorhandenen Drucksteigerung erwähnt wird, so scheint mir der Grund darin zu liegen, dass das Exsudat meist nur in mässiger Menge vorhanden ist.

Bei dem Umstande, dass die serösen Höhlen des menschlichen Körpers, namentlich nach der operativen Eröffnung derselben, so häufig entzündlichen Erkrankungen unterliegen, muss es gewiss als auffallend erscheinen, dass die Tenon'sche Kapsel nur selten entzündlich afficirt wird. Besonders muss hervorgehoben werden, dass nach der operativen Eröffnung der 'Tenon'schen Kapsel bei Schieloperationen, Tenonitis, wie oben erwähnt, nur ausnahmsweise beobachtet wird.

Cohnheim giebt an, dass die Disposition eines Organes, entzündlich zu erkranken, mit seinem Reichthume an Gefässen zusammenhängt. Gewiss ist diese Erklärung auch für die Seltenheit der Entzündung der Tenon'schen Kapsel zutreffend, da dieselbe, soweit die unvollkommenen Untersuchungen über dieselbe zu schliessen gestatten, nur spärlich mit Gefässen versehen ist.

Erwähnen will ich noch, dass ich bei einer Anzahl von an Iridocyclitis erkrankten Augen stellenweise Infil-

*) Graefe u. Saemisch Handbuch II., p. 372.

**) Manometrische Bestimmung des intraoculären Druckes. Centralbl. f. d. med. Wissensch., No. 36. 1866. 
tration des mit dem Bulbus innig verbundenen inneren Blattes der Tenon'schen Kapsel mit Rundzellen beobachtete.

\section{Anatomische Untersuchung eines Falles von Cataracta ossea.}

In der älteren ophthalmologischen Literatur finden sich Beschreibungen einer Anzahl von Fällen, in welchen eine „Verknöcherung” der Kristall-Linse beobachtet wurde, doch scheint es, dass in diesen Füllen keine strenge Scheidung zwischen der Verkalkung und der Verknöcherung der Linse gemacht wurde.

Der erste Fall, in welchem an Stelle der Linse echte Knochensubstanz mikroscopisch nachgewiesen wurde, ist von R. Wagner*) beschrieben. "Unter anderen interessanten Dingen fand ich kürzlich in dem atrophischen Auge einer männlichen Leiche aus den mittleren Lebensjahren eine glatte, scheibenförmige, steinharte Linse von $2 \mathrm{Mm}$. Dicke, die ich mit Herrn Dr. Schrader näher untersuchte. Wir fanden dieselbe ganz in echte Knochensubstanz verwandelt, mit den schönsten vielstrahligen Knochenkörperchen (d.h. Knochenhöhlen und Knochencanälchen) und von Havers'schen Markcanälen durchzogen. Von der ursprünglichen Linsensubstanz war, so weit die jetzt noch nicht vollendete Untersuchung ergab, keine Spur mehr nachzuweisen". Ueber das Verhalten der Knochenneubildung zur Linsenkapsel spricht sich Wagner nicht aus.

Lohmeyer**), welcher das obige Präparat gesehen zu haben scheint, giebt an, dass die Knochenneubildung innerhalb der Linsenkapsel liege.

Es ist der Fall von R. Wagner aus theoretischen Gründen vielfach angefochten worden. Die Entwicklungsgeschichte lehrt bekanntlich, dass nach der Trennung der

*) Veränderungen thierischer Gewebe in morphologischer und. chemischer Beziehung. Nachr. d. kgl. Gesellsch. d. Wissensch. za Göttingen 1851. No. 8.

**) Beiträge zur Histiologie und Aetiologie der erworbenen Linsenstaare. Zeitschr. f. rat. Med., V. Bd. Hft. 1 u. 2, p. 92. 
Keimblätter, die Bestandtheile des einen Keimblattes sich nicht mehr in die eines anderen Keimblattes umwandeln können, welche Thatsache zuerst von Thiersch und Waldeyer auch für die Pathologie als giltig anerkannt wurde und mit einer Ausnahme (Sticker*) auch gegenwärtig allgemein für richtig angesehen wird.

Es wurde als unmöglich erllärt, dass aus den Linsenfasern, welche aus dem Ektoderm stammen, sich Knochen bilden könne, welches letztere Gewebe dem Mesoderm angebört.

Virchow**) erwähnt den Fall R. Wagner's bei der Besprechung des Osteoms des Glaskörpers. Virchow glaubt, dass bei vollständigem Schwunde der Linse Knochenbildung im Glaskörper aufgetreten sei. In ähnlicher Weise spricht sich H. Müller ***) aus.

Pagenstecher $\dagger$ ), welcher in einem Falle von Netzhautablösung. Knochenbildung im Glaskörper fand, glaubt ebenfalls, das es sich bei Wagner's Fall um eine analoge Erscheinung handle.

Knapp $\dagger$ †) leugnet die Möglichkeit von wahrer Knochenbildung in der Kristall-Linse. Nach seiner Anschauung gehen alle Knochenbildungen im Auge blos von der Choriocapillaris aus.

v. Stellwag $\dagger \dagger \dagger$ ) hält seit dem Jahre 1853 an der in seinem Werke, die Ophthalmologie rom naturwissenschaftlichem Standpunkte" und in sümmtlichen 5 Auflagen seines Lehrbuches ausgesprochenen Ansicht fest, dass Knochenbildung in der Linse vorkomme. „Den thatsächlichen Beobachtungen gegenüber fallen die Bedenken, welche man in nenerer Zeit betreffs der Möglichkeit einer Ossification der Linse erhoben hat (Virchow, Pagenstecher) nicht allauschwer ins Gewicht".

Bei Gelegenheit der Kapselcataracte führt H. Müller (ges. und hinterlassene Schriften, I. p. 289) eine ähnliche Ansicht von Förster an: „Prof. Förster hat die Bemerkung gemacht, dass im Falle in der That die Masse, welche sich p. 453.

*) Vorlesungen über allgem. u. experiment. Pathologie. 1878.

**) Die krankhaften Geschwülste, II. Berlin 1864. p. 101.

***) Ges. u. hinterlass. Schriften. p. 137.

†) Arch. f. Ophthalm. VII. 1, p. 115, 117.

††) Archiv f. Augen- und Ohrenheilkunde II. 1, p. 133.

$+\dagger \dagger)$ Lehrbuch der prakt. Augenheilk. 5. Auf., p. 673.

v. Graefe's Archiv fuir Ophthalmologie, XXIX. 4. 
innen an der Kapsel entwickelt, für gleichwerthig mit einer Bindegewebssubstanz gehalten werden dürfte, es auch nicht unmöglich scheine, dass darin einmal eine knochenartige Substanz zur Ausbildung komme".

Berthold*) bescheibt zwei Fälle, bei welchen sich an der Stelle der Linse echtes Knochengewebe von der Linse entsprechender Form und Grösse fand. In den Markräumen lagen (p. 107) „Pigmentkiumpen und Pigmentlörnchen eingeschwemmt und zwar am stärksten an den Stellen, welche der vorderen Fläche der Linse zunächst lagen, weiter nach dem Innern der Linse fand sich weniger Pigment in den Markränmen. Berthold fasst diese Fälle als Knochenbildung in der Iris auf und will dies ,aus der Form der Ablagerung von Pigment in den Markräumen erweisen" (p. 111).

v. Becker **) sagt: „Selbst, wenn die von Wagner beobachtete Knochenbildung innerhalb der Kapsel gelegen hat, wäre es weiter von Wichtigkeit zu wissen, ob sich die Knochensubstanz innerhalb der unverletzten Knochensubstanz entwickelt hatte. Es liegt auf der Hand, dass wir nur in diesem Falle von einer Verknöcherung der Linse sprechen können. War die Kapsel verletzt, so würde eine Knochenneubildung deshalb nichts Auffallendes haben, weil wir wissen, dass sich überall aus Bindegewebe Knochen bilden kann".

Den gleichen Standpunkt hält v. Becker in seinem vor Kurzem erschienenen Werke ,Zur Anatomie der gesunden und kranken Linse" aufrecht (p. 141). In Betreff der oben angeführten Ansicht Förster's sagt v. Becker (p. 79): „Weil die Kapselcataract bei unverletzter Kapsel niemals verknöchert, wird es um so unwahrscheinlicher, dass die Substanz Bindegewebe ist".

Dass Cataracta ossea bei intacter Linsenkapsel ebenso wenig denkbar ist, als dies für die Cataracta purulenta und fibrosa bereits vielfach erwiesen ist, ergiebt sich bereits aus dem Umstande, weil der Knochen ein reiches Gefässsystem besitzt, welches mit den Blutgefässen des Auges communiciren muss.

*) Beiträge zur pathologischen Anatomie des Auges. Arch. f. Ophthalm. XVIIII. 1, p. 104.

**) Graefe und Saemisch Handbuch V, p. 194. 
Goldzieher*) beschreibt einen Fall von Knochenbildung in eyclitischen Schwarten, welche die Linse umgeben. Es ,.mag dieser Fall als Beweis dienen, dass die Linse als solche eine knöcherne Schale erhalten kann, welche fähig ist, sich nach innen durch Apposition zu verdicken und, da Nährmaterial in überreicher Quantität zugeführt wird, nach dem Schwunde der Linsenfaseru auch ein massiver knöcherner Körper mit vollständiger Linsengestalt sich bilden kann." Goldzieher meint, dass es sich in manchen als Cataracta ossea beschriebenen Fällen um eine ähnliche Erscheinung handeln mag.

Ferner beschreibt Goldzieher einen Knochenkern, welcher seiner Lage und Form nach vollständig der Linse entspricht. Von der Linsenkapsel war nichts mehr nachweisbar. Goldzieher glaubt, dass die letztere durch Usur zu Grunde gegangen sein möge (l. c. p. 332).

Arlt**) hat zwei Fälle von Knochenbildung innerhalb der Linsenkapsel gesehen. In einem Falle (p. 211) waren „Binderewebe, Kalk- und Knochengewebe, das völlig die Structur des normalen zeigt, neben einander innerhalb der Linsenkapsel." In dem zweiten Falle (Verletzung) war neben ausgedehnten Knochenbildungen in der Aderhaut ,der ganze Inhalt der gefalteten in cyclitisches Gewebe eingeschlossenen Linsenkapsel Knochengewebe."

Ayres ***) beschreibt ferner einen Fall von Cataracta ossea. Die Linsenkapsel war nicht gefaltet. Zwischen der letzteren und dem Knochen war eine schmale Zone von Bindegewebe nachweisbar. An einer Stelle der Linsenkapsel konnte eine Perforation erkannt werden.

In der Literatur finde ich noch folgende, wie es scheint, meist zweifelhafte Fälle von Knochenbildung in der Linse:

Canton, Ossification du cristallin et de la capsula. Lancet und Annal. d'Oculist. XXIX, p. 51, 1853. (Wahrscheinlich Verkalkung.)

*) Die Verknöcherungen im Auge. Arch. f. Augenheilk. IX. 1880 , p. 332.

**) Compendium der normalen und der pathologischen Histologie des Auges. 1880.

***) Ueber Knochenbildung in der Linsenkapsel. Arch. für Augenheilk. XI. 3, p. 327. 
France J., Cas d'ossification et de déplacement de la lentille cristalline. Gaz. méd. de Paris No. 4, 1846, Ann. d'Oculist. XV, p. 38.

Gluge, Note sur l'ossification du cristallin. Ann. d'Ocul. X, p. 226.1843.

Hunt, Ossification du cristallin. Americ. Journ., Juill. p. 94.1864.

Kirk, Depôts osseux dans la membrane vitreuse et le cristallin. Month. Journ. Nov. 1853.

Magne, Cataracte eapsulaire ossifiée, passée dans la chambre antérieure; extraction. Annal. d'Ocul. XVIII, p. 271 (wahrscheinlich Verkalkung). 1847.

Sichel, Cas rare d'ossification de la capsule cristalline dans une cataracte traumatique. Ann. d'Ocul. XI, p.223, 1844.

Durch die besondere Güte des Herrn Dr. W. Uhthoff, Assistenten an der Augenklinik des Herrn Prof. Schöler in Berlin gelangte ich in den Besitz einer Anzahl von phthisischen Bulbis, welche wegen drohender sympathischer Krkrankung des anderen Auges enueleirt wurden. Bei einem derselben konnte ich das Bestehen von sogenannter Cataracta ossea beobachten. Da die Anzahl der genauer beschriebenen derartigen Fälle eine geringe ist, mag es gerechtfertigt erscheinen, wenn ich die Resultate der anatomischen Untersuchung dieses Auges veröffentliche.

Der Bulbus ist auf nahezu Hasselnussgrösse geschrumpft. Die Hornhaut lässt folgende Veränderungen erkenuen. Das Epithel ist ungleichmassig verdickt mit zahlreichen papillenartigen Fortsätzen an dessen hinteren Fläche versehen. Gleiche papilläre Wucherungen zeigt das Epithel der Conjunctiva bulbi. Das Hornhautparenchym ist von Gefässen durchsetzt, welche in der Nähe der Oberfläche besonders zahlreich erscheinen (Vergl. Fig. 1). Im Hornhautparençhym finden sich in der Umgebung der Gefässe streckenweise Finlagerungen von Rundzellen. 
Die Descemet'sche Membran ist stark gefaltet, rnit der hinter derselben gelegenen bindegewebigen Schwarte, welche die vordere Fläche der Iris bedeckt, verwachsen. Nur an einzelnen Stellen lassen sich bei mikroskopischer Untersuchung Lüeken zwischen der M. Descemeti und dieser Schwarte erkennen, welche mit Endothelzellen ausgebildet sind. Es sind diese Lücken mit Wahrscheinlichkeit als Reste der vorderen Kammer anzusehen.

An einer Stelle ist die Descemet'sche Membran in ihrer Continuität getrennt, ein Umstand, der allein auf eine vorhergegangene Perforation derselben schliessen lässt.

Ein fernerer Umstand, der für eine besondere Perforation spricht, ist in dem unregelmässigen Baue, welchen der der Continuitätstrennung der Descemet'schen Membran entsprechende Theil des Hornhautparenchyms zeigt, begründet.

Faserzüge (n), welche mit den die Vorderkammer erfüllenden Schwarten zusimmenhängen, durchsetzen in schiefer Richtung einen Theil der Hornhaut.

Die Sclerotica ist an einzelnen Stellen von gruppenweise angeordneten Rundzellen infiltrirt.

Die Iris ist an ihrer vorderen Fläche mit einer bindegewebigen Sccwarte bedeckt. Die Iris selbst ist verdickt und mit Rundzellen durchsetzt. An ihrer hinteren Fläche finden sich papillare Wucherungen, welche mit den cyclitischen Schwarten an der Vorderfläche der Linse verwachsen sind.

Die Ciliarfortsätze sind beträchtlich proliferirt. Der Accomodationsmuskel ist verdickt. Die Muskelbündel desselben sind auseinander gedrängt, zwischen denselben finden sich bindegewebige Fasern und Rundzellen.

Die Muskelbündel selbst zeigen hochgradige Veränderungen. Die Zellen sind verbreitert: ihr Protoplasma tingirt sich mit Carminammoniak und Haematoxylin sehr 
intensiv, was bekanntlich bei organischen Muskelfasern nicht der Fall ist.

Die Anzahl der Kerne ist vermehrt. Es sind dieselben zumeist in Reihen hinter einander angeordnet. Meist sind deren 2-3 in einer Zelle vorhanden, doch habe ich in einzelnen bis zu 8 Kernen gefunden. Die Contouren der Muskelfasern zeigen sich an jenen Stellen, an welchen die Kerne in denselben liegen, leicht verbreitert.

Das Verhalten der Kerne gegen Reagentien weicht ebenfalls vom normalen ab, indem sich dieselben mit Carminammoniak und Haematoxylin bedeutend schwächer tingiren, als das Protoplasma. Die Form der Kerne ist eine rundliche bis ovale.

Zwischen den Kernen erscheinen an Zupfpräparaten die Muskelzellen von queren Linien durchsetzt, so dass es den Anschein hat, als würden die Muskelfasern in hinter einander geordnete Stücke zerfallen.

Es erinnern diese Bilder an ähnliche Erscheinungen bei der parenchymatősen Entzündung der quergestreiften Muskelfasern.*)

Es sind diese Vorgänge als entzändliche Wucherung des Accomodationsmuskels aufzufassen.

Gussenbauer **) führt an, dass die glatten Muskelfasern in Carcinomen proliferiren. Durante ***) berichtet über das Proliferiren der glatten Muskelfasern in den Blutgefässen bei auf experimentellem Wege hervorgerufener Entzündung derselben.

Die Pigmentschichte des Ciliarkörpers (Fig. 1, p s) ist beträahtlich verbreitert. Durch Wucherung der Zellen der Pars ciliaris retinae ( $p$ e r) ist dieselbe auf das 10-20-

*) Vergl. Birch-Hirschfeld, Lehrb. d. patholog. Anatomie 1877, p. 321 und Rindfleisch, Patholog. Gewebelehre 1878, p. 635.

**) v. Langenbeck's Archiv. Bd. IV.

***) Wiener med. Jahrb. 1871. 
fache verbreitert. Kolbige Fortsätze (Anlagen neugebildeter Gefässe) gehen von ihr in die cyclitischen Schwarten.

Die Form und die Lage der Linse nimmt eine Knochenmasse (Fig. 1, k) ein mit deutlichen Knochenkörperchen, Havers'schen Lamellen, Canälen und Markräumen.

An der vorderen Fläche derselben lässt sich deutlich die stark gefaltete und verdeckte vordere Linsenkapsel ( $\mathrm{kk}$ ) erkennen, die am Querschnitte bei stărkeren Vergrösserungen längs gestreift erscheint.

Der Stelle der Verletzung der Hornhaut entsprechend (n) ist auch die vordere Kapsel in ihrer Continuität getrennt und sehr stark eingerollt, ein Umstand, der ebenfalls dafür spricht, dass die Linsenkapsel verletzt worden war.

Entsprechend dieser von der vorderen Linsenkapsel nicht bedeckten Stelle des Knochens finde ich an Anzahl von Schnitten Blutgefässe, weche aus den vor der Knochenmasse gelegenen cyclitischen Schwarten in dieselbe hineinziehen.

Die Fasern der Zonula lassen sich deutlich erkennen; im aequatorialen Theile der Linse lässt sich an zerzupften Präparaten der Uebergang derselben in die Linsenkapsel leicht feststellen.

Die hintere Kapsel ist an den meisten Präparaten nicht nachweisbar, bloss kleinere Theile derselben finden sich erhalten.

So finde ich an einem in Fig. 2 abgebildeten Schnitte einen am Querschnitte schlingenförmig erscheinenden Theil der hinteren Kapsel ( $h \mathrm{k}$ ) erhalten, dessen beide Endtheile von der Knochensubstanz umwachsen erscheinen.

Es ist sehr leicht verständlich, dass die hintere Kapsel, welche viel zarter ist, als die vordere, dem Zugrundegehen durch Resorption nicht lange Widerstand leistet. 
Im aequatorialen Theile des Knochens finde ich zwischen demselben und der Linsenkapsel eine grössere Ansammlung von Bindegewebe. An der vorderen Fläche ist meist nur eine zarte bindegewebige Schichte zwischen dem Knochengewebe und der Linsenkapsel, an einzelnen Stellen berührt der Knochen dieselbe, ja es erscheinen Falten desselben vom Knochengewebe umwachsen. Ebenso finde ich meist zwischen den Resten der Hinterkapsel und dem Knochen eine bindegewebige Schichte.

Es muss die Resistenz der Linsenkapsel, besonders der vorderen Kapsel, hervorgehoben werden, namentlich, wenn man bedenkt, dass, wie zahlreiche experimentelle Untersuchungen an Thieren ergaben, in den Knochen eingetriebene glatte Elfenbeinstäbchen durch lacunäre Resorption nach einiger Zeit eine rauhe Oberfläche zeigten.*)

An denjenigen Stellen, an welchen die vordere Kapsel mit der Oberfläche des Knochens in Berührung steht, zeigt die dem letzteren zugewandte Fläche der ersteren kleine grubige Vertiefungen. Es scheinen dieselben dafür zu sprechen, dass die Linsenkapsel ebenso, wie Elfenbeinstifte, welche in den Knochen eingestossen werden, der lacunären Resorption unterliegt, doch sind diese grubigen Vertiefungen an der Linsenkapsel kein constanter Befund.

Bei der Färbung mit Carminammoniak oder Haematoxylin zeigte dies innerhalb der Linsenkapsel entstandene Knochengewebe folgende Erscheinung, die besonders an stärker tingirten Präparaten (vergl. Fig. 2) sehr auffüllig hervortritt. Die Oberfläche des in Müller'scher Elüssigkeit entkalkten Knochens ist durch das sich bei obiger Behandlung entwickelnde Chromoxyd grün gefärbt

*) Arlt (l. c.) bildet einen Schnitt durch eine Cataracta ossea $a b$, bei welchem die Knochenmasse die Linsenkapsel vollständig erfüllt, nirgends von derselben durch Bindegewebe getrennt erscheint, die Linsenkapsel aber vollständig erhalten sein soll. 
( $0 \mathrm{k}$ ), während der central gelegene Theil des Knochens sich roth resp. violett färbt. Allein auch in der Umgebung der Havers'schen Canäle und der Markräume finden sich ringförmige Zonen, welche sich mit Tinctionsmitteln nicht färben. In Fig. 2. finden wir einen Markraum abgebildet, im Centrum desselben ein grösseres Gefäss und Markzellen. Die Peripherie des Markraumes nimmt eine Schichte von grossen Zellen mit deutlichen runden Kernen ein $(0 \mathrm{~s})$. Es sind dies Osteoblasten. Hierauf findet sich eine schmale Schichte, in welcher die Knochentextur sehr. dentlich hervortritt ( $\mathrm{n} k$ ). Es ist dies neugebildeter, unverkalkter Knochen, der mit Carminammoniak sich sehr stark imbibirt.

Hierauf erscheint, durch eine scharfe Grenze vom vorigen getrennt, eine ringförmige grün gefärbte Partie, (ok) die mit Tinctionsmitteln sich nicht imbibirt; es ist dies kalkhaltiger Knochen. Das nach aussen von der mit $0 \mathrm{k}$ bezeichneten ringförmige Stelle liegende Knochengewebe (f k) färbt sich ebenso intensiv, wie die unverkalkten Theile des Knochens.

Bekanntlich tingirt sich nach den Beobachtungen von Frey*) das der Knochensalze beraubte (entkalkte) Knochengewebe mit Färbemitteln ebenso intensiv, als das unverkalkte und zwar kann man, wie von verschiedenen anderen Beobachtern hervorgehoben wurde, durch verschieden lange Kalkentziehung alle Nuancen von der schwächsten bis zur stärksten Färbung erhalten.

Es musste daher die Frage entschieden werden, $a b$ die central gelagerten Theile des Knochens (Fig. 2, $\mathrm{fk}$ ), bekanntlich die älteren Bestandtheile des Knochengewebes, kalklos oder minler kalkhaltig als die später entwickelten Theile des Knochens sind. 1873. p. 183. 
Bemerken will ich noch, dass die Grenzen zwischen dem grün gefärbten nnd den mit Tinctionsmitteln gefärbten centralen Knochengebieten in den meisten Fällen diffus sind, selten durch eine scharfe Grenzlinie von einander getrennt erscheinen, während kalkhaltiger und kalkloser Knochen stets durch scharfe Grenzen von einander getrennt erscheint.

Es hat obige Frage einiges Interesse mit Rücksicht auf die Art und Weise, in welcher im physiologischen Knochen die Kalksalze abgelagert werden. Im neugebildeten Knochen werden nämlich in die von den Gefassen am weitesten abstehenden Theilen zuerst, wie dies von Thierfelder*), Schottelius**) u. A. nachgewiesen wurde, die Kalksalze abgelagert. Es entspricht dies der allgemeinen Erfahrung, dass zum Zustandekommen der Verkalkung eines Gewebes eine Herabsetzung der Ernährung desselben nothwendig ist.

Thierfelder ***) sagt: „Je weiter ein Gewebe von den Canälen der Nahrungszufuhr abliegt, um so träger wird es von der Ernährungsflüssigkeit durchströmt und um so günstigere mechanische oder chemische Bedingungen bietet es für Ablagerung von Kalksalzen dar."

Um nun zu entscheiden ob die centralen Theile des Knochens entkalkt oder unverkalkt sind, wurden Präparate, welche die Untersehiede mit Carminammoniak sehr dentlich zeigten, in Kali aceticum $\dagger$ ) entfärbt. Es kann an solchen Präparaten dann deutlich entschieden werden, welche Partien entkalkt sind, indem dieselben weniger

*) Ueber die Bedeutung der provisorischen Knorpelverkalkung. Arch. der Heilk. XVI, 1875. p. 441.

**) Die Kehlkopfknorpel. Wiesbaden 1879. p. 34.

***) ]. c. p. 446.

†) Vergl. Pommer, Ueber die lacunäre Resorption in erkrankten Knochen. Wiener Sitz.-Ber. LXXXVIII. 3. Abth. Jan.Heft 1881. p. 63. 
deutlich die Knochenstructur erkennen lassen, als die unverkalkten.

Es zeigte sich nun, dass die mit Carmin nicht tingirten Theile des Knochens weniger deutlich die Structur erkennen liessen als die durch Apposition neu entstandenen kalklosen Theile desselben.

Es war jedoch auch zwischen dem ungefärbten Theile (ok) und dem gefürbten Theile $(\mathrm{fk})$ ein auffallender Unterschied erkennbar. Letzterer liess minder deutlich die Structur erkennen, als ersterer, ist mithin jedenfalls verkalkt gewesen.

Die in der Umgebung der Markräume und an der Oberfläche des Knochens gelegenen Stellen waren jedoch vielfach mit Kalkkrümmelchen durchsetzt. Die Knochenstructur war in denselben undeutlicher erkennbar, als in den mit fk bezeichneten Theilen des Körpers.

Die Vermuthung liegt nahe, dass diese Erscheinung darauf zurückzuführen ist, dass die später angelagerten Knochenpartieen einen stärkeren Kalkgehalt besitzen, als diejenigen, welche sich früher gebildet haben.

Es wird Sache der pathologischen Histologie sein, diese Frage weiter zu verfolgen.

Bemerken will ich noch, dass ich an einer Anzahl von Knochenneubildungen in der Aderhaut von phtisischen Augen eine ähnliche Erscheinung beobachten konnte.

In der Literatur finde ich die Beobachtung einer ähnlichen Erscheinung von Pommer*) erwähnt, welche derselbe als ,unvollständige diffuse Verkalkung” bezeichnet.

An zahlreichen Stellen an der Knochenoberfläche lassen sich Auflagerungen von neugebildeten Knochen er-

*) Ueber dic Osteoklaestentheorie. Virchow's Arch., 92. Bd. 1883 , p. 311. P. findet die Knochensubstanz bei obiger Veränderung, die er selten gefunden hat, ,von gleichmässiger gewöhnlich sehr undeutlich fibrillärer Beschaffenheit und nur dadurch von der 
kennen. Auf dem Querschnitte fällt der Reichthum an Howship'schen Lacunen und Kittlinien auf. Es sind diese Befunde ein Beweis dafür, dass der pathologisch neugebildete Knochen denselben Umwandlungen dureh Apposition und Resorption unterworfen ist, wie der physiologische.*)

Ein Befund scheint mir dafür zu sprechen, dass der durch Apposition vergrösserte Knochen, nachdem er die Grenze der hinteren Kapsel überschritten hat, sich weiter in die cyclitischen Schwarten ausdehnen kann. An einzelnen Präparaten finde ich nämlich, dass ein zapfenartiger Fortsatz vom äquatorialen Rande der Linse eine Strecke. weit bis in die Nähe des Glaskörpers zieht; die äussere Begrenzung dieser Knochenmasse bildet die Zonula Zinnii.

Der Glaskörper ist beträchtlich geschrumpft, an seiner hinteren Oberfläche von der trichterförmig abgehobenen Netzhaut bedeckt. Der Glaskörper ist in ein maschenförmiges Bindegew ebe mit reichlichen eingelagerten Kernen umgewandelt. Die Gefässe desselben stammen zum Theile aus dem Corpus ciliare, theils aus dem Sehnerven und der Netzhaut. Die Gefässwandungen, sowie zahlreiche narbenähnliche Faserzüge (Fig. $3 \mathrm{na}$ ), welche den Glaskörper durchsetzen, färben sich mit Carmin oder Hämatoxylin minder intensiv als ersterer.

Erwähnen will ich noch einiger spaltenförmiger Hohlräume (Fig. $3 \mathrm{gs}$ ), welche im Glaskörper vorkommen. Es haben dieselben eine unregelmässige Form und zeigen scharfe Begrenzungslinien. Ein Endothel konnte ich an

vollständig homogen verkalkten Umgebung, in welche dieselbe ohne deutliche sichtbare Grenze übergeht, unterschieden, dass sie in Carmin eine blassviolette oder rosenrothe Färbung annahm". Die "homogen und vollständig verkalkten" Stellen sind ungefärbt, die gänzlich kalklosen „intensiv kirschroth".

*) Vergl. v. Ebner, Ueber den feineren Bau der Knochen. substanz. Wiener Sitz.-Ber. 72. Bd. 1875 und Pommer, 1. c. 
der Innenwand derselben nicht nachweisen. Der Inhalt derselben besteht aus einer lymphartigen Flüssigkeit, in welcher farblose und vereinzelte farbige Blutkörperchen sich finden. Vacuolenartige Gebilde (va) in dieser Flüssigkeit dürften wohl nur als Kunstproduct aufzufassen sein.

Die Frage nach der Bedeutung dieser spaltenförmigen Räume muss ich vorlänfig offen lassen; da der Nachweis eines Endothels nicht gelungen ist, bin ich nicht berechtigt, dieselben als erweiterte Lymphgefässe aufzufassen.

Die abgehobene Netzhaut (Fig. 1 ne) ist bindegewebig entartet. Dadurch, dass der Accomodationsmuskel Erscheinungen von entzündlicher Wucherung des Gewebes darbietet, ferner der vordere Theil des Bulbus beträchtlich geschrumpft ist, erscheint die abgeloste Netzhaut fast in einer Linie mit dem hinteren Theile des Accommodationsmuskels gezerrt. Zwischen der Aderhaut und der Netzhaut bestehen abnorme Gefässverbindungen. Eine Anzahl von nengebildeten Gefässen zieht aus dem vordersten Theile der Aderhaut, von Pigment umscheidet, in die Netzhaut. In der Netzhaut selbst ist ein Knochenkern mit deutlichen Havers'schen Canälen und Lamellen zu beobachten. Im Centrum desselben findet sich ein weiter Markraum, der von Fettzellen erfüllt ist und einzelne Gefässe und Pigmentzellen enthält. Ein von der Aderhaut stammendes Gefäss (Fig. $1 \mathrm{gf}$ ) lässt sich bis in diesen Markraum hinein verfolgen.

In der Umgebung der Blutgefässe der Netzhaut finden sich sternförmige Pigmentzellen abgelagert.

Zwischen der Aderhaut und der Netzhaut findet sich ein Exsudat abgelagert, in welchem Rundzellen und Pigmentzellen abgelagert sind. Die innere Oberfläche der Aderhaut lässt Glasdrusen (c d) in grösserer Menge erkennen.

Der Sehnerv zeigt eine beträchtliche Verdickung des bindegewebigen Balkenwerkes, namentlich in der Lamina 
cribrosa. An einzelnen Stellen finden sich Anhäufungen von Rundzellen in den Scheiden und in dem Intervaginalraume des Sehnerven. In der Umgebung der Centralgefässe des Sehnerven sind an einzelnen Stellen ebenfalls Anhäufungen von Rundzellen erkennbar.

Die Aderhaut erscheint im hintere Theile des Auges durch massenhafte Bildung von Bindegewebe verdickt. In der Umgebung des Sehnerven ist ein Knochenkern in der Aderhant eingelagert, dessen zahlreiche Markräume mit Fettzellen erfullt sind und Pigmentzellen enthalten.

Der Vollständigkeit halber will ich noch die Befunde an den Ciliarnerven mittheilen.

Bevor die Ciliarnerven in die Hornhaut eintreten, verlaufen sie eine Strecke weit in der Sclerotica. Ich finde daselbst Infiltration der Scheiden mit Rundzellen und massenhafte Vermehrung der Kerne zwischen den Nervenfasern. Zwischen letzteren finden sich ebenfalls Rundzellen eingelagert. Einzelne Axencylinder sind an umschriebenen Stellen beträchtlich verdickt, sclerosirt.

Im anderen Theile des Suprachorioidalraumes ist die Infiltration der Nerven und ihrer Scheiden eine viel beträchtlichere als an obigen Stellen.

In den Canälen, in welchen die Ciliarnerven den hinteren Theil der Sclerotica durchziehen, zeigen einzelne entzündliche Veränderungen, während ich sie an anderen Nervenbündeln vermisste.

In Betreff der Knochenbildung innerhalb der Linsenkapsel ergiebt sich in diesem Falle folgendes:

Die Besehaffenheit des Hornhautparenchyms, die Continuitätstrennung der M. Descemeti und die der letzteren entsprechende Unterbrechung der anderen Kapsel lassen auf eine stattgefundene Verletzung mit Perforation der vorderen Kapsel schliessen. 
Die Knochenbildung ist von der vorderen Kapsel an den meisten Stellen noch dureh Bindegewebe getrennt. Die hintere Kapsel ist mit Ausnahme einzelner Stellen, welche ihre frühere Lage andeuten, durch Resorption zu Grunde gegangen.

Wenn wir die verschiedenen Erklärungen für das Zustandekommen dieser Knochenneubildung durchgehen, so müssen wir zunächst von der Möglichkeit, dass dieselben von eingewanderten Osteoblasten herrühren, abstrahiren, da nicht abzusehen ist, auf welchem Wege dieselben in den Bulbus gelangt sein sollten. Busch behauptet nämlich, dass überall dort, wo Knochengewebe sich neubildet, dies nur durch Wanderung von Osteoblasten zu Stande komme.

Die Annahme, dass die Knochenneubildung von der vom mittleren Keimblatte abstammenden Linsenkapsel nach v. Becker*) gehört bloss die äussere Lamella derselben dem Mesoderm an, während die inneren Schichten entsprechend der Auffassung Kölliker's cuticulare Bildungen sind - ausgehe, ist ebenfalls unberechtigt. Es liegen keine Beobachtungen vor, dass Knochengewebe von Glashäuten ausgehe, mit Ausnahme einer Mittheilung Alt's**), der eine Knochenneubildung als von der Glashaut der Chorioidea ausgehend beschreibt.

Diese Beobachtungen von Alt müssen jedoch schon aus dem Grunde in Zweifel gezogen werden, weil Knochengewebe nur in vascularisirten Geweben entstehen kann. Der Umwandlung des Knorpels in Knochen geht bekanntlich die Neubildung von Gefässen voraus und selbst in vascularisirten Geweben ist vor dem Eintritte der Ossification eine Vermehrung der Gefăsse zu beobachten, wie dies z. B. Kassowitz***) an den Sehnen der Vögel

*) Zur Anatomie der gesunden und kranken Linse. p. 88.

**) l. c. p. 130.

***) Wiener Med. Jahrb. 1879. p. 179. 
nachwies. Bisher steht jedoch der Beweis noch aus, dass in structurlosen Häuten sich Gefässe bilden können.

Man kann ferner ebenfalls nicht annehmen, dass diese Knochenneubildung von der Iris ausgegangen sei, da in der letzteren, ebenso wie in dem Ciliarkörper, niemals Knochenneubildung beobachtet wurde.

Es bleibt mithin nur die eine Möglichkeit bestehen, dass die Knochenneubildung von dem Bindegewebe einer Cataracta fibrosa a usgegangen sei, für welche Annahme, wie oben erwähnt wurde, v. Becker zuerst eingetreten ist.

Da übersichtliche Darstellungen über das Vorkommen von Knochenneubildung im Auge fehlen, will ich hier eine kurze Uebersicht derselben geben. Es kommen letztere vor:

1. In der Bindehaut. Loring (The New York med. Journ. XXXVII., p. 59), A. Crittchett. — Es entspricht dieses Osteon den Hautknochen (Virchow).

2. Im subconjunctivalen Gewebe. v. Graefe, Zehender's Klin. M-Bl. 1863. p. 23, Saemisch in Graefe und Saemisch Handbuch IV, p. 151.

3. In der Sclerotica. Watson, Transact of the patholog. Soc. of London. Vol. XXII. p. 227. Es entspricht diese Knochenbildung den Osteomen der Dura Mater und den Sehnenknochen.

4. In der Aderhaut. Analog diesen Knochenbildungen sind die Osteome der Arachnoidea des Gehirns und des Rückenmarks.

5. In der abgelösten Netzhaut. (v. Sehiess, Goldzieher u. A.). Diesen Knochenbildungen entsprechen umschriebene Verknöcherungsheerđ̉e des Gehirns (Sims, Benjamin, Ebstein).

6. Im bindegewebig entarteten Glaskörper. v. Wittich, Arch. f. pathologische Anatomie 1853, V. 
p. 580. - Virchow, Die krankh. Geschwülste II, p. 100 (bei Pferden). - Poncet, Wecker in Graefe und Sämisch's Handbuch IV, p. 719. - v. Becker, Klin. M.-Bl. 1871, p. 428. - Pagenstecher 1. c.

7. In den cyclitischen Schwarten in der Omgebung der Linse (Knapp, Schiess, Goldzieher und von Anderen).

8. In einer Cataracta fibrosa.

Erklärung der Abbildungen.

Fig. 1. Meridionalsehnitt durch den vorderen Abschnitt. des Auges. Carminfärbung. Merz, System No. 1.

b d Bindegewebige Schwarten in der Umgebung der Linse.

c Conjunctiva.

c d Glasdrüsen der Aderhaut.

c e Hornhautepithel.

c p Hornhautparenchym.

c s In Narbengewebe umgewandelte cyclit. Schwarten.

g f Blutgefäss.

g k Glaskörper.

k Knochengewebe.

md Descemet'sche Membran.

n Narbengewebe in der Hornhaut.

ne Netzhaut.

per Pars ciliaris retinae.

ps Pigmentschichte des Ciliarkörpers.

s Sclerotica.

vk Vordere Linsenkapsel.

Fig. 2. Hinterer Theil der Cataracta ossea. Meridionalschnitt. Carminfärbung. Merz, System No. II (System III zum Zeichnen der Details verwendet).

c s Cyclitische Schwarten.

fk Entkalkte Knochen, stark tingirt.

hk Hintere Linsenkapsel (ein schlingenförmiges Stück derselben).

g f Blutgefäss.

v. Graefe's Archiv für Ophthalmologie, XXIX. 4. 
k 1 Kittlinie,

n k Unverkalkter Knochen, stark tingirt.

o k Kalkhaltiger Knochen, nugefärbt.

o s Osteoblasten.

Fig. 3. Glaskörper an einem Meridionalsehnitte durch den vorderen Theil des Auges. Carmin-Hämatoxylinfärbung. Merz, System No. III.

$\mathrm{g} f$ Blutgefässs.

gk Kernreiches Glaskörpergewebe.

g s Spaltenartiger Raum im Glaskörper.

n a Narbenzüge im Glaskörper.

v a Vacuolenartige Gebilde (Kunstproducte). 